Gynecologic and

Obstetric Investigation
Received: March 8, 2012

Accepted after revision: June 3, 2012

Published online: August 25, 2012

\title{
Effect of Umbilical Cord Bleeding following Mid-Pregnancy Cordocentesis on Pregnancy Outcomes
}

\author{
Theera Tongsong Raveewan Khumpho Chanane Wanapirak \\ Wirawit Piyamongkol Supatra Sirichotiyakul \\ Department of Obstetrics and Gynecology, Faculty of Medicine, Chiang Mai University, Chiang Mai, Thailand
}

\section{Key Words}

Fetal outcomes · Prenatal diagnosis · Cordocentesis

\begin{abstract}
Objectives: To compare the adverse pregnancy outcomes among the three groups of umbilical cord bleeding following cordocentesis; a group of no bleeding (group 1), transient bleeding (group 2), and persistent bleeding (>60 s: group 3). Methods: Consecutive cases of cordocentesis were prospectively recorded and followed up. The inclusion criteria for analysis consisted of (1) singleton pregnancies; (2) gestational age of 18-22 weeks; (3) no fetal anomalies; (4) no previous invasive procedures; (5) performed by experienced operators, and (6) known pregnancy outcomes. The main outcomes were rates of fetal loss, low birth weight and preterm birth. Results: Of 2,174 procedures, 1,614 were in group 1, 509 were in group 2, and 51 were in group 3. The mean birth weight and gestational age at delivery were significantly lower in group 3 than those in groups 1 and 2. The fetal loss rate was significantly higher in group 3 . Rates of low birth weight and preterm birth were significantly higher in group 3 whereas the rates in group 2 had a tendency to be higher than those in group 1. Conclusion: This study suggests that cord bleeding during cordocentesis carries a higher risk of fetal loss, low birth weight and preterm birth.
\end{abstract}

\section{KARGER}

Fax +4161306 1234

E-Mail karger@karger.ch

www.karger.com (c) 2012 S. Karger AG, Basel

0378-7346/12/0744-0298\$38.00/0

Accessible online at:

www.karger.com/goi

\section{Introduction}

Cordocentesis or funipuncture is a prenatal diagnostic procedure for fetal blood sampling which was first performed under ultrasound guidance by Daffos et al. [1] in 1980. This prenatal procedure has been well accepted as an efficient and relatively safe procedure and has permitted new insight into fetal medicine. A variety of indications for cordocentesis have been reported, especially rapid karyotype, fetal hemolytic disease and severe, early-onset growth restriction as well as fetal infection [2-6]. Unlike in the Western countries, cordocentesis in our practice for more than 20 years is most commonly indicated for fetal diagnosis of severe thalassemia syndrome, followed by rapid karyotype $[7,8]$. However, this procedure carries a significant risk of pregnancy complications [8-13]. Though the rate of complications following cordocentesis has been ardently discussed in the literature, only a few studies have addressed this question comprehensively. Cordocentesis-related fetal loss is presumably associated with several factors, such as prolonged bleeding from the puncture site, formation of umbilical cord hematoma, premature rupture of membranes, etc. $[9,10$, $14,15]$. Umbilical cord bleeding which is commonly observed following cordocentesis is often linked to subsequent serious complications even fetal loss. However, only inconclusive data are available on the clinical sig- 
nificance of umbilical cord bleeding following cordocentesis. No study has specifically focused on such a relationship. The data in previous studies are heterogeneous and those studies are flawed by several uncontrolled confounders such as mixed gestational age, various levels of expertise of the performers, and the presence of placenta penetration, etc. Thus, this study aimed to compare the adverse pregnancy outcomes among the three groups of umbilical cord bleeding secondary to cordocentesis - no bleeding, transient bleeding and prolonged bleeding - after excluding or controlling for potential confounding factors on adverse pregnancy outcomes.

\section{Materials and Methods}

A prospective study to compare adverse pregnancy outcomes between cordocentesis with cord bleeding and without bleeding was undertaken from a single center, Maharaj Nakorn Chiang Mai Hospital, Chiang Mai, Thailand, between September 1989 and December 2010. During the study period all cases of cordocentesis were prospectively recorded for procedure details as well as baseline characteristics of the patients, such as gestational age, indications for cordocentesis. The procedure profiles were also immediately recorded during the procedure such as the puncture cord site, puncture with/without placenta penetration, the presence of umbilical cord bleeding as well as its duration, bradycardia and duration of the procedure time, etc. All pregnancies were followed up for final pregnancy outcomes. These data were stored in a computer for subsequent analysis. The inclusion criteria for analysis consisted of (1) singleton pregnancies; (2) gestational age of 18-22 weeks at the time of performing cordocentesis; (3) no fetal congenital anomalies; (4) pregnancies with previous cordocentesis (usually from other hospitals or previous failure), or amniocentesis or chorionic villous sampling in the current pregnancy; (5) the procedures performed by experienced operators, referred to those experienced of more than 50 procedures, and (6) known pregnancy outcomes. The exclusion criteria were (1) the procedures involving placenta penetration; (2) fetuses subsequently proven to have chromosomal or structural abnormalities or any serious disorders required termination of pregnancy, and (3) failed or difficult procedures (procedures with duration time $>30 \mathrm{~min}$.) All procedures were performed under transabdominal-ultrasound guidance, using the convex transducer of $3.5 \mathrm{MHz}$ (model E-8; GE Medical Systems, Milwaukee, Wisc., USA, and model SSD 620, 680EX, SSD 1700; SSD 5000, alpha-10; Aloka, Tokyo, Japan).

We used the same techniques in all cordocenteses, freehand technique, using local anesthesia without prophylactic antibiotics and without fetal paralysis to eliminate fetal movement. Puncture site selection was based on accessibility and quality of visualization with an attempt to avoid placenta penetration, if possible. The details of techniques are described elsewhere [7]. The failed procedures were based on operators' decisions. There was no definite number of punctures or exact duration of procedure, though a procedure lasting $>30$ min was considered a failure in most cases. The pregnancies with normal results of fetal blood analysis were usually taken care of as routine antenatal care.

Cord Bleeding following Cordocentesis
Cases meeting the inclusion criteria were divided into three groups as follows: group 1: no bleeding; group 2: transient bleeding defined by bleeding from the umbilical cord puncture $<1 \mathrm{~min}$, and group 3: cordocentesis with prolonged bleeding, defined by bleeding from the umbilical cord puncture $>1 \mathrm{~min}$.

The primary outcomes of this study consisted of rates of fetal loss, low birth weight and preterm birth, as well as rate fetal bradycardia. Fetal loss was defined by spontaneous abortion (before 20 weeks of gestation) or a dead fetus in utero of any gestational age, and neonatal death. Low birth weight was defined by infants with a birth weight $<2,500 \mathrm{~g}$. Preterm birth referred to delivery before 37 complete weeks of gestation. This research was approved by the research ethics committee of our institute.

\section{Statistical Analysis}

Pearson's $\chi^{2}$ test was used to compare the qualitative data or rates of adverse outcomes such as fetal loss rates among the three groups, whereas the ANOVA test with the post-hoc test were used for comparison of the quantitative data like means of gestational age at delivery or birth weight. The test results were considered statistically significant at $\mathrm{p}<0.05$. Statistical analyses were performed using the software for the social sciences (SPSS-PC+) software package version 17.0 (SPSS, Inc., Chicago, Ill., USA).

\section{Results}

During the study period, a total of 6,147 cordocenteses were performed at 18-22 weeks of gestation. To reduce as many potential confounding factors as possible and make the available data homogeneous, 3,973 cases were excluded due to the following reasons: twin pregnancies (55), loss to follow-up/incomplete data, especially final outcomes of pregnancies $(1,272)$, procedures undergoing during early learning curve of experience $(1,123)$, cases with prior cordocentesis/amniocentesis or chorionic villous samplings (866), failed or difficult procedures (40), fetuses later proven to have structural or chromosomal abnormalities or serious disorders required for termination of pregnancy (802), and procedures with placenta penetration (615). Finally, 2,174 procedures were suitable for analysis. Of these, 1,614 (74.2\%) procedures had no cord bleeding (group 1), 509 (23.4\%) had transient cord bleeding ( $\leq 60 \mathrm{~s}$ ) (group 2) and the remaining $51(2.4 \%)$ had prolonged cord bleeding (>60 s) (group 3). Indications for cordocentesis are presented in table 1. Distribution of percentages of the indications was comparable among the three groups. Fetal risk for severe thalassemia and higher risk for chromosomal abnormalities was the most common indication. Distribution of percentages of placental location was also comparable in the three groups, as shown in table 2 . The means of maternal age among the three groups were not significantly different 
Table 1. Indications for cordocentesis

\begin{tabular}{|c|c|c|c|c|c|c|}
\hline & \multicolumn{2}{|c|}{ Group 1} & \multicolumn{2}{|c|}{ Group 2} & \multicolumn{2}{|c|}{ Group 3} \\
\hline & $\mathrm{n}$ & $\%$ & $\mathrm{n}$ & $\%$ & $\mathrm{n}$ & $\%$ \\
\hline Risk of fetal Hb Bart's disease & 312 & 19.3 & 94 & 18.5 & 13 & 25.5 \\
\hline Risk of fetal $\beta$-thalassemia major & 210 & 13.0 & 66 & 13.0 & 9 & 17.6 \\
\hline Risk of fetal $\beta$-thalassemia/HbE & 435 & 27.0 & 142 & 27.9 & 8 & 15.7 \\
\hline Mixed risk of fetal severe thalassemia & 14 & 0.9 & 5 & 1.0 & 0 & 0.0 \\
\hline Rapid karyotyping & 372 & 23.0 & 103 & 20.2 & 11 & 21.6 \\
\hline Risk of fetal severe thalassemia + chromosome & 262 & 16.2 & 97 & 19.1 & 8 & 15.7 \\
\hline Others & 9 & 0.6 & 2 & 0.4 & 2 & 3.9 \\
\hline Total & 1,614 & 100.0 & 509 & 100.0 & 51 & 100.0 \\
\hline
\end{tabular}

Table 2. Placental location

\begin{tabular}{|c|c|c|c|c|c|c|}
\hline & \multicolumn{2}{|c|}{ Group 1} & \multicolumn{2}{|c|}{ Group 2} & \multicolumn{2}{|c|}{ Group 3} \\
\hline & $\mathrm{n}$ & $\%$ & $\mathrm{n}$ & $\%$ & $\mathrm{n}$ & $\%$ \\
\hline Anterior & 452 & 28.0 & 146 & 28.7 & 12 & 23.5 \\
\hline Anterior lateral & 125 & 7.7 & 23 & 4.5 & 3 & 5.9 \\
\hline Anteroposterior & 17 & 1.1 & 7 & 1.4 & 1 & 2.0 \\
\hline Posterior & 915 & 56.7 & 288 & 56.6 & 32 & 62.7 \\
\hline Posterior lateral & 25 & 1.5 & 3 & 0.6 & 0 & 0.0 \\
\hline Lateral & 19 & 1.2 & 3 & 0.6 & 1 & 2.0 \\
\hline Posterior fundus & 4 & 0.2 & 5 & 1.0 & 0 & 0.0 \\
\hline Fundus & 56 & 3.5 & 34 & 6.7 & 2 & 3.9 \\
\hline Unspecified & 1 & 0.1 & 0 & 0.0 & 0 & 0.0 \\
\hline Total & 1,614 & 100.0 & 509 & 100.0 & 51 & 100.0 \\
\hline
\end{tabular}

$\chi^{2}$ test: there were no significant differences between the three groups.

$(30.78 \pm 7.51$ vs. $30.52 \pm 8.1$ vs. $30.44 \pm 6.9$ years; F test; $\mathrm{p}=0.62$ ). The procedure-related complications and outcomes of pregnancy outcomes were presented in table 3.

Considering technical aspects, the mean duration to complete the procedure in group 3 was not significantly different (ANOVA test, $p<0.001$ ). Likewise, the rates of the difficult procedure and successful rates were not significantly different among the three groups $\left(\chi^{2}, \mathrm{p}>0.05\right)$. However, the rates of fetal bradycardia were significantly higher in the prolonged bleeding group (3.3 vs. 4.1 and $15.7 \%$ in groups $1-3$ respectively, $\mathrm{p}<0.001)$. Regarding adverse outcomes of pregnancy, the mean birth weight and gestational age at delivery were also significantly lower in group 3 than those in groups 1 and 2, respectively, as shown in table 3 . The fetal loss rate in group 3 was significantly higher when compared to that in groups
1 and 2, and the rate in group 2 was also significantly higher than that in group 1 (1.6 vs. 4.1 and $11.8 \%$ in group 1-3 respectively, $\mathrm{p}<0.001)$. Likewise, low birth weight rates and preterm birth rates were significantly higher in group 3 ( $\mathrm{p}<0.001)$, whereas the rates in group 2 had a tendency to be higher than those in group 1, but were not statistically significant.

\section{Discussion}

Transient bleeding from the needle puncture site is a common finding often lasting no more than $1 \mathrm{~min}$. The prevalence in this study was approximately $25 \%$ which was nearly the same as the occurrence we observed in our previous report, $20 \%$ for transient bleeding and 5\% for 
Table 3. Comparisons of procedure and pregnancy outcomes among the three groups (prolonged bleeding, transient bleeding, no bleeding)

\begin{tabular}{|c|c|c|c|c|c|c|c|}
\hline & \multicolumn{2}{|l|}{ Group 1} & \multicolumn{2}{|l|}{ Group 2} & \multicolumn{2}{|c|}{ Group 3} & \multirow[t]{2}{*}{$\mathrm{p}$ value } \\
\hline & mean & SD & mean & $\mathrm{SD}$ & mean & SD & \\
\hline Procedure time & 5.48 & 5.89 & 5.37 & 5.43 & 7.08 & 8.22 & $0.219^{*}$ \\
\hline Birth weight & 3,003 & 503 & 2,998 & 592 & 2,541 & 870 & $<0.001^{* *}$ \\
\hline \multirow[t]{3}{*}{ GA at delivery } & 38.2 & 2.2 & 37.9 & 2.7 & 36.2 & 4.7 & $<0.001^{* *}$ \\
\hline & \multicolumn{2}{|l|}{ Group 1} & \multicolumn{2}{|l|}{ Group 2} & \multicolumn{2}{|c|}{ Group 3} & $\mathrm{p}$ value \\
\hline & $\mathrm{n}$ & $\%$ & $\mathrm{n}$ & $\%$ & $\mathrm{n}$ & $\%$ & \\
\hline Success rate & $1,598 / 1,612$ & 99.1 & $500 / 507$ & 98.6 & $51 / 51$ & 100.0 & 0.457 \\
\hline Bradycardia & $53 / 1,614$ & 3.3 & $21 / 509$ & 4.1 & $8 / 51$ & 15.7 & $<0.001$ \\
\hline Fetal loss & $26 / 1,614$ & 1.6 & $21 / 509$ & 4.1 & $6 / 51$ & 11.8 & $<0.001^{\#}$ \\
\hline Low birth weight & $185 / 1,614$ & 11.5 & $62 / 509$ & 12.2 & $14 / 51$ & 27.5 & $<0.001^{\# \#}$ \\
\hline Preterm birth & $195 / 1,614$ & 12.1 & $70 / 509$ & 13.8 & $14 / 51$ & 27.5 & $<0.001^{\# \#}$ \\
\hline
\end{tabular}

* ANOVA test shows no significant mean differences among the three groups.

** Post hoc test shows that the pairs of significant mean difference is group 3-group 2 and group 1-group 3.

\# $\chi^{2}$ test: significant difference between group 3-groups $1 \& 2$ and group 1 -group 2.

\# Significant difference between group 3-groups $1 \& 2$ (note that there are unsuccessful cases including contamination of maternal blood or laboratory failure in diagnosis, etc.)

prolonged bleeding [7]. This is also comparable with that reported by Liao et al. [10], who found a prevalence of $19.8 \%$. Our results indicate that transient bleeding has a tendency to increase the rate of adverse outcomes while prolonged bleeding is strongly associated with such outcomes. Bleeding from the cord puncture site is visible and is usually monitored to cessation and recorded for bleeding duration. Several factors may be associated with umbilical cord bleeding, especially difficult procedures, thick maternal abdominal wall, not the easiest accessible site to target, poor visualization, and active fetal movement. Active fetal movement while the needle tip is in lumen of the umbilical vessels is likely to increase risk of injury to those vessels, leading to bleeding.

Several authors believe that umbilical cord bleeding during the procedures carries a higher risk of adverse pregnancy outcomes, but no strong evidence has confirmed this hypothesis before, although several cases of fetal compromise have been reported to be associated with cord bleeding. For example, Maeda et al. [13] reported that 1 case in their series of 43 cases had continuous bleeding for $11 \mathrm{~min}$ and ended up undergoing cesarean section due to fetal distress. Many studies $[9,12-14,16-$ 19] have been published regarding cordocentesis-related complications, but most did not have enough statistical power to show the real effect of umbilical cord bleeding on pregnancy adverse outcomes of low prevalence, especially fetal loss rates. Additionally, previous studies are flawed by heterogeneous baseline characteristics leading to be unsuitable for comparison.

To be more perfectly compared between those groups, we tried to eliminate all known or potential confounding factors which might have affected adverse outcomes of the procedures to achieve high homogeneity of all baseline characteristics in the three groups. The potential confounders needed to be controlled or excluded, included placenta traversed, difficult procedures, twin pregnancies, gestational age, and levels of operators' experience. Placenta traversed has been associated with adverse outcomes [20]. Gestational age certainly has an influence on the outcomes of procedures and pregnancy. Therefore, all cordocenteses included in this study were only confined to mid-pregnancy, i.e. a gestational age of 18-22 weeks. Our previous studies suggest that the procedures during the early learning curve usually take more time to complete, implying an increase in adverse outcomes of pregnancies [7]. Thereby, all of the procedures in the first 50 cases of practice were excluded from this study to reduce the expertise effect on pregnancy outcomes. Moreover, all difficult cases defined by the procedure duration 
time of $>30$ min were also excluded. It makes sense to assume that difficult procedures or long operations are likely to increase fetal risk either with or without umbilical cord bleeding, thus confounding the results. Theoretically, the procedures involving penetration of the placenta are likely to increase the risk of trauma to placental vasculatures, leading to fetal or maternal hemorrhage, either bleeding into amniotic fluid or subtle bleeding in the placenta. This certainly can cause fetal anemia or thrombosis or placental ischemia, or even result in abortion or subsequent preterm labor. Thus, procedures with placenta penetration were excluded from our analysis.

Fetal bradycardia, immediate complications during the procedure, was significantly increased with duration of umbilical cord bleeding. It is reasonable to assume that umbilical cord bleeding can cause fetal bradycardia, implying fetal compromise and possibly leading to fetal loss. In addition to the fetal loss rate, rates of preterm birth rate and low birth weight were also slightly, but significantly higher in the group of cord bleeding, consistent with lower mean birth weight and mean gestational age. These complications may be associated with fetal anemia or some thrombosis with possibly impeding fetal circulation. Note that the rate of procedure time was comparable in all groups since the difficult cases were excluded.

It is theoretically reasonable to assume that more bleeding time is likely to be associated with a higher rate of adverse pregnancy outcomes. Whether such a transient bleeding, which is one of the most common findings during operation, can have any true effects on adverse outcomes or not has not be determined before. This study strongly suggests that any umbilical cord bleeding should not be ignored and the performers encouraged to avoid any condition leading to umbilical cord bleeding, e.g. not making an attempt to puncture during fetal active movement, temporary discontinuation of the procedures during bleeding, and no puncture with poor visualization. We recommend that in case of excessive fetal movement compromising access to the ideal cord site or placing an increased risk to the fetus, performers should wait for a while or even postpone the procedure until several hours later. Using a smaller needle, a 25 -gauge needle, may be helpful for less blood loss [21].

Some pitfalls in this study may exist. The procedurerelated fetal loss could not be accurately evaluated. The fetal loss rate in this study included a background risk. Some authors define the procedure-related fetal loss to be that it occurs within 2 weeks of gestation. Unfortunately, we could not reliably analyze the effect on such a specific fetal loss since the number of fetal losses was too low to gain adequate power for such a purpose. Additionally, some confounding factors such as types of punctured vessels or the number of needle insertions could not be exactly provided for analysis in this study. Finally, the long period of study associated with considerable changes in operators, loss to follow-up rates as well as advancement in ultrasound equipment may affect the outcomes of the procedure. However, such changes were unlikely to alter the results since they should be the same among the groups of bleeding.

The strength of this study was the large sample size from a single center which was highly homogeneous and gained enough power to assess the rare outcomes like fetal loss rate. The effects of known confounders were controlled, especially the expertise levels of performers and similar gestational age. Besides, some highly potential confounders to outcomes of measurement were excluded as often as possible, such as the procedures with placenta penetration, difficult cases, and twin pregnancy, etc. Therefore the interpretation of the results may probably be highly reliable.

In conclusion, this study suggests that umbilical cord bleeding during cordocentesis carries a higher risk of fetal loss, low birth weight and preterm birth, and it seems to be related to the duration of bleeding. This information may encourage the performers to avoid any condition leading to umbilical cord bleeding.

\section{Acknowledgements}

The authors wish to thank Thai Research Fund and the National Research University Project under Thailand's Office of the Higher Education Commission for financial support.

\section{Disclosure Statement}

The authors have no conflicts of interest to disclose.

References A new procedure for fetal blood sampling in utero: preliminary results of fifty-three cases. Am J Obstet Gynecol 1983;146:985-987.

$\checkmark 2$ Donner C, Rypens F, Paquet V, et al: Cordocentesis for rapid karyotype: 421 consecutive cases. Fetal Diagn Ther 1995;10:192-199.

-3 Mathur R, Dubey S, Hamilton S, et al: Rapid prenatal karyotyping using foetal blood obtained by cordocentesis. Natl Med J India 2002;15:75-77. 
4 Megerian G, Ludomirsky A: Role of cordocentesis in perinatal medicine. Curr Opin Obstet Gynecol 1994;6:30-35.

$\checkmark 5$ Nicolaides KH, Thilaganathan B, Mibashan RS: Cordocentesis in the investigation of fetal erythropoiesis. Am J Obstet Gynecol 1989;161:1197-1200.

6 Weiner CP: Cordocentesis for diagnostic indications: two years' experience. Obstet Gynecol 1987;70:664-668.

$>7$ Tongsong T, Wanapirak C, Kunavikatikul C, et al: Cordocentesis at 16-24 weeks of gestation: experience of 1,320 cases. Prenat Diagn 2000;20:224-228.

$>8$ Tongsong T, Wanapirak C, Kunavikatikul C, et al: Fetal loss rate associated with cordocentesis at midgestation. Am J Obstet Gynecol 2001;184:719-723.
$>9$ Ghidini A, Sepulveda W, Lockwood CJ, Romero R: Complications of fetal blood sampling. Am J Obstet Gynecol 1993;168:13391344.

10 Liao C, Wei J, Li Q, et al: Efficacy and safety of cordocentesis for prenatal diagnosis. Int J Gynaecol Obstet 2006;93:13-17.

11 Whittle MF: The safety of cordocentesis. Br J Hosp Med 1989;41:511.

12 Weiner CP, Okamura K: Diagnostic fetal blood sampling-technique related losses. Fe tal Diagn Ther 1996;11:169-175.

13 Maeda H, Shimokawa H, Satoh S, et al: Safety of cordocentesis under ultrasound guidance for fetal blood sampling. Nippon Sanka Fujinka Gakkai Zasshi 1990;42:199-202.

14 Atkins AF: Safety in numbers for cordocentesis. Br J Hosp Med 1989;42:510.

15 Acar A, Balci O, Gezginc K, et al: Evaluation of the results of cordocentesis. Taiwan J Obstet Gynecol 2007;46:405-409.
6 Antsaklis A, Daskalakis G, Papantoniou N, Michalas S: Fetal blood sampling - indication-related losses. Prenat Diagn 1998;18: 934-940.

7 Bernaschek G, Yildiz A, Kolankaya A, et al: Complications of cordocentesis in high-risk pregnancies: effects on fetal loss or preterm delivery. Prenat Diagn 1995;15:995-1000.

18 Daffos F: Fetal blood sampling. Annu Rev Med 1989;40:319-329.

19 Petrikovsky B: Complications after cordocentesis appear excessive. Am J Obstet Gynecol 1997; 177:1567.

20 Boupaijit K, Wanapirak C, Piyamongkol W, et al: Effect of placenta penetration during cordocentesis at mid-pregnancy on fetal outcomes. Prenat Diagn 2012;32:83-87.

21 Kawakami Y, Matsuda H, Shibasaki T, et al: Safer cordocentesis by new 25 -gauge needles. Fetal Diagn Ther 2008;24:211-217. 